\title{
Emerging roles of eosinophils and eosinophil-derived lipid mediators in the resolution of inflammation
}

\author{
Yosuke Isobe ${ }^{1}$, Taiga Kato ${ }^{1}$ and Makoto Arita ${ }^{1,2 *}$ \\ ${ }^{1}$ Department of Health Chemistry, Graduate School of Pharmaceutical Sciences, University of Tokyo, Tokyo, Japan \\ 2 Precursory Research for Embryonic Science and Technology, Japan Science and Technology Agency, Saitama, Japan
}

\section{Edited by:}

Janos G. Filep, University of

Montreal, Canada

Reviewed by:

Ralf J. Ludwig, University of Lübeck, Germany

Takayuki Yoshimoto, Tokyo Medical

University, Japan

*Correspondence:

Makoto Arita, Department of Health

Chemistry, Graduate School of

Pharmaceutical Sciences, University

of Tokyo, 7-3-1 Hongo, Bunkyo-ku,

Tokyo 113-0033, Japan.

e-mail:marita@mol.f.u-tokyo.ac.jp
Acute inflammation and its resolution are essential processes for tissue protection and homeostasis. Once thought to be a passive process, the resolution of inflammation is now shown to involve active biochemical programs that enable inflamed tissues to return to homeostasis. The mechanisms by which acute inflammation is resolved are of interest, and research in recent years has uncovered new endogenous anti-inflammatory and proresolving lipid mediators (i.e., lipoxins, resolvins, protectin, and maresin) generated from polyunsaturated fatty acids (PUFAs). This review presents new insights into the cellular and molecular mechanisms of inflammatory resolution, especially the roles of eosinophils, and a series of omega-3 PUFA-derived anti-inflammatory lipid mediators that they generate.

Keywords: resolution of inflammation, lipid mediator, eosinophils, lipidomics, lipoxygenase, resolvins

\section{INTRODUCTION}

Inflammation is a defensive response to injury and infection, but excessive or inappropriate inflammation contributes to a range of acute and chronic human diseases. Acute local inflammation in healthy individuals is self-limited and resolves by means of an active termination program (Gilroy et al., 2004; Serhan and Savill, 2005). The mechanisms that regulate the progression and resolution of inflammation remain of interest. Lipid mediator metabolomics of self-resolving inflammatory exudates recently uncovered a new family of potent anti-inflammatory and pro-resolving mediators. These include arachidonic acid (AA)-derived lipoxins, eicosapentaenoic acid (EPA)-derived E-series resolvins, docosahexaenoic acid (DHA)derived D-series resolvins, protectin, and maresin (Bannenberg and Serhan, 2010). In inflammatory exudates, lipid mediators change in the course of acute inflammation and resolution (Levy et al., 2001; Bannenberg et al., 2005; Blaho et al., 2009; Yamada et al., 2011). In this review, we provide an overview of the novel cellular and molecular components involved in lipid mediator class switching during resolution of inflammation, especially eosinophils and the lipid mediators that they generate.

\section{INFLAMMATORY RESPONSE AND THE RESOLUTION OF INFLAMMATION}

Inflammation is a host defense mechanism that is characterized by the movement of serum proteins and leukocytes from the blood to the extravascular tissue. The acute inflammatory response is characterized by the initial recruitment of neutrophils, followed by the recruitment of monocytes that differentiate into macrophages (Figure 1). Many mediators coordinate the initial events of acute inflammation. Lipid mediators such as prostaglandins (PGs) and leukotrienes (LTs), cytokines, and chemokines coordinately regulate vascular permeability and leukocytes infiltration (Larsen and Henson, 1983). Once the noxious materials are removed via phagocytosis, the inflammatory reaction must be resolved to maintain homeostasis. The resolution of acute inflammation is an active process that is controlled by endogenous pro-resolving mediators. These factors switch off leukocyte trafficking to the inflamed site, reverse vasodilation, and vascular permeability, and promote the clearance of inflammatory cells, exudates, and tissue debris, thereby leading to the restoration of homeostasis to the inflamed tissue. AA-derived lipoxin $\mathrm{A}_{4}\left(\mathrm{LXA}_{4}\right)$ was the first PUFA-derived mediator found to have anti-inflammatory and/or pro-resolving activities (Godson et al., 2000; Serhan, 2005). Nanomolar concentrations of $\mathrm{LXA}_{4}$ inhibit polymorphonuclear leukocyte (PMN) entry into inflamed tissues in animal models (Colgan et al., 1993). $\mathrm{LXA}_{4}$ is synthesized by the actions of both 15-lipoxygenase (15LOX) and 5-LOX, and phosphorylation of 5-LOX at S663 was recently shown to convert the enzyme to a robust 15-LOX, which can stimulate production of $\mathrm{LXA}_{4}$ in cells which do not express 15-LOX (Gilbert et al., 2012). Also, omega-3 fatty acids EPA and DHA are precursors of endogenous anti-inflammatory and/or pro-resolving mediators. Using an unbiased lipidomics approach and the enzymatic oxygenation of omega-3 fatty acids, Serhan and collaborators identified families of novel bioactive mediators derived from EPA and DHA. These include EPA-derived E-series resolvin (RvE1, E2; Serhan et al., 2000; Arita et al., 2005; Tjonahen et al., 2006), DHA-derived D-series resolvin (RvD1-6; Serhan et al., 2002; Sun et al., 2007; Spite et al., 2009; Chiang et al., 2012), neuroprotectin/protectin (NPD1/PD1; Hong et al., 2003; Marcheselli et al., 2003; Serhan et al., 2006), and maresin (MaR1; Serhan et al., 2009, 2012). These lipid mediators promote resolution via enhanced macrophage clearance of apoptotic PMNs, chemokines, cytokines, and microbial products (Ariel et al., 2006; Schwab et al., 2007). In the course of acute inflammation and 


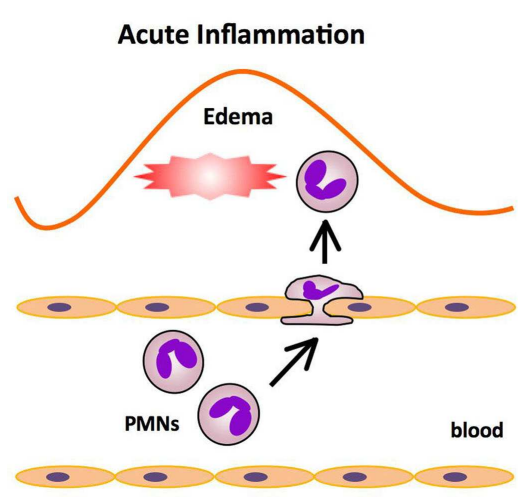

FIGURE 1 | Major processes in acute inflammation and resolution. The initiation phase of acute inflammation is characterized by the rapid infiltration of polymorphonuclear neutrophils (PMNs) followed by the infiltration of monocytes that mature into macrophages, and edema formation in response to injury. PMNs provide the first line of immune

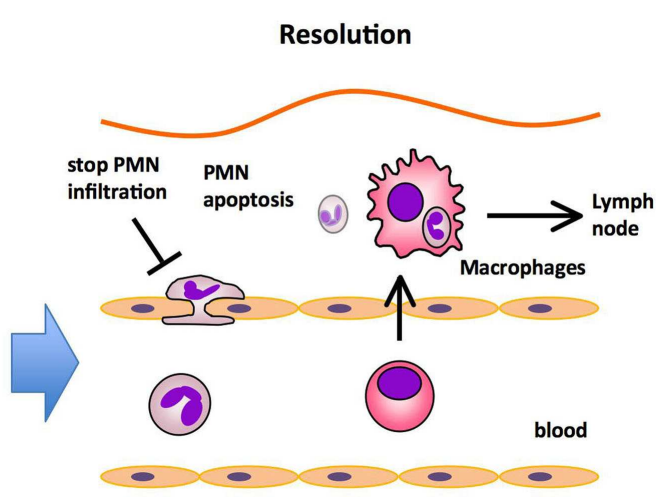

defense by migrating to sites of injury and neutralizing invading microorganisms or noxious materials by phagocytosis. In the resolution phase, PMNs undergo apoptosis and are ingested by macrophages that emigrate rapidly from the inflamed site to the draining lymph nodes (DLNs).
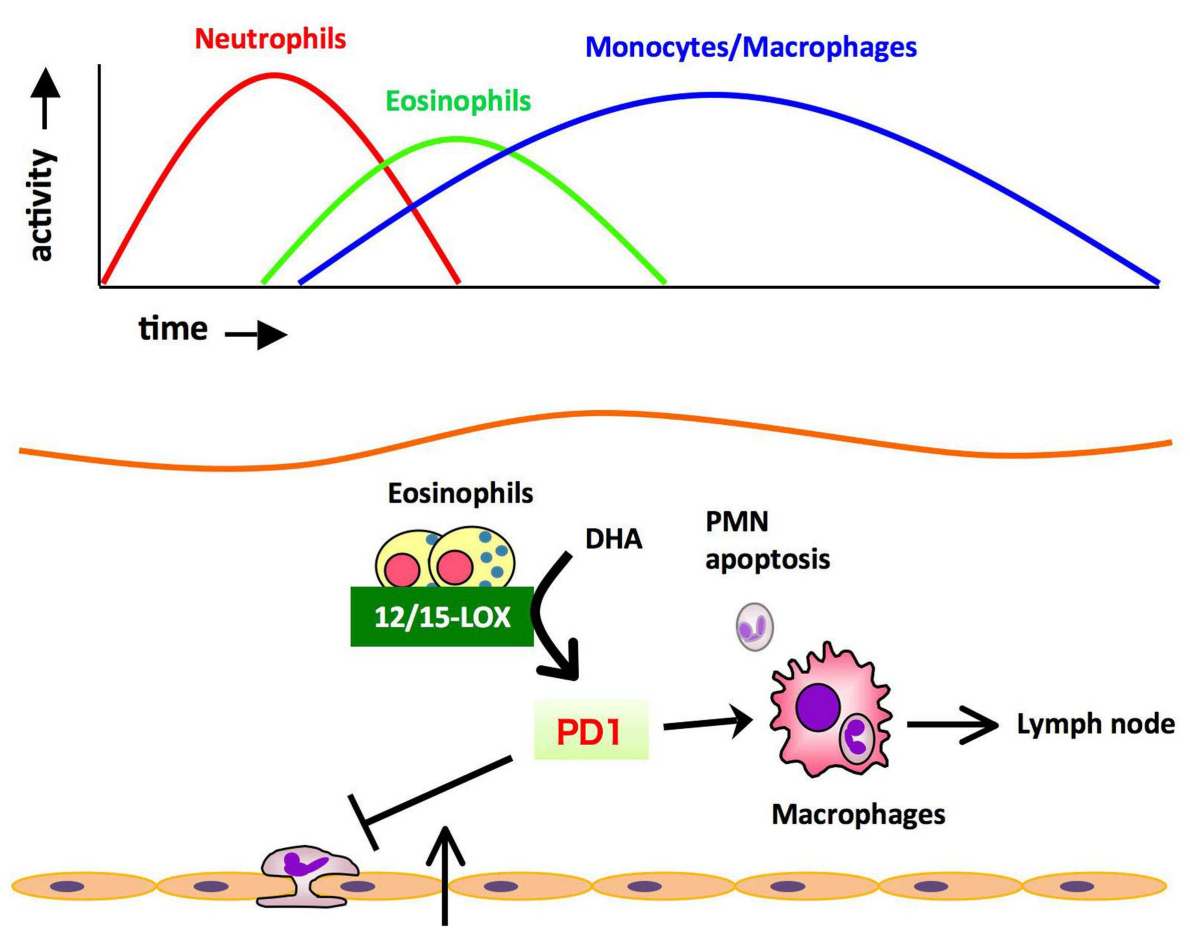

PMNs

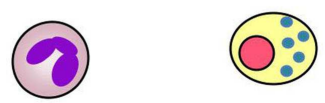

blood

FIGURE 2 | Eosinophils are recruited to the inflamed loci and promote resolution of inflammation by producing anti-inflammatory and pro-resolving lipid mediators. During the resolution phase of self-resolving acute peritonitis model, eosinophils are recruited to the inflammatory site, where they locally produce pro-resolving lipid mediators such as PD1 via 12/15-LOX-initiated biosynthesis. These mediators block PMN infiltration and/or promote the clearance of phagocytes carrying engulfed zymosan from the inflammatory site to DLNs. resolution, changes of cellular composition or cell-cell interactions are accompanied by a switch of lipid mediator profiles in exudates (Serhan, 1997, 2007). The temporal switch in lipid mediator class is an active process that they underscore the ability of inflammatory cells to trigger the self-limited response of acute inflammation. 


\section{NOVEL ROLES OF EOSINOPHILS IN PROMOTING THE RESOLUTION OF ACUTE INFLAMMATION}

The profile of lipid mediators during inflammation-resolution was determined with a liquid chromatography-tandem mass spectrometry (LC-MS/MS)-based lipidomic analysis. In murine zymosan-induced peritonitis, the maximal levels of 5-LOX products such as leukotriene $\mathrm{B}_{4}\left(\mathrm{LTB}_{4}\right)$ were observed in the initiation phase, and subsequently decreased during the resolution. In comparison, the levels of 12/15-LOX products such as protectin D1 (PD1) were low at the initiation of inflammation, then gradually increased during the resolution phase. The DHA-derived lipid mediator PD1 is biosynthesized via a $12 / 15$-LOX-mediated pathway that converts DHA into a 10,17-dihydroxy-containing bioactive molecule (Hong et al., 2003; Marcheselli et al., 2003; Serhan et al., 2006). PD1 promotes resolution by counter regulating PMN influx and stimulating macrophage ingestion of apoptotic PMNs, and increasing phagocyte clearance into DLNs (Schwab et al., 2007). Therefore, the major cellular components of PD1 biosynthesis in the resolution phase were of interest. Recently we identified eosinophils as major PD1-producing cells in the resolution phase of zymosan-induced peritonitis (Yamada et al., 2011). Eosinophils are multifunctional leukocytes that have been implicated in the pathogenesis of numerous inflammatory processes, including parasitic infections and allergic diseases. However, the roles of eosinophils in acute inflammation and resolution are unclear. To determine the role of eosinophils in the resolution of inflammation, an anti-IL-5 monoclonal antibody (Corry et al., 1996) was administered to deplete eosinophils from mice challenged with zymosan. In vivo depletion of eosinophils resulted in an increased number of PMNs and a reduced number of phagocytes leaving the inflamed peritoneum to the draining lymph nodes (DLNs), both of which showed a resolution deficit. The LC-MS/MS-based lipidomic analysis revealed that the amounts of 12/15-LOX-derived mediators including PD1 were dramatically decreased in eosinophil-depleted mice, whereas the amounts of COX and 5-LOX derived products did not differ between the two groups. Adoptive transfer of wild type eosinophils, but not eosinophils from 12/15-LOX knockout mice, successfully restored the resolution phenotype. Also, administration of PD1 restored the resolution phenotype. These results indicate that eosinophils are recruited to the inflamed loci during the resolution phase, where they locally produce anti-inflammatory and pro-resolving lipid mediators such as PD1 via a 12/15-LOX-initiated biosynthetic route, which contribute to resolution (Figure 2 ).

Eosinophils are circulating granulocytes that typically mature in the bone marrow, and can be recruited to sites of immunological or inflammatory responses. Triggering of eosinophils by engagement of receptors for cytokines, immunoglobulins, or complement can lead to the secretion of an array of cytokines [IL-2, IL-4, IL-5, IL-10, IL-12, IL-13, IL-16, IL-18, and transforming growth factors (TGF- $\alpha / \beta)$ ], chemokines (RANTES and eotaxin-1), lipid mediators [platelet-activating factor (PAF), and leukotriene $\mathrm{C}_{4}\left(\mathrm{LTC}_{4}\right)$ ], and cytotoxic granule cationic proteins [major basic protein (MBP), eosinophil peroxidase (EPO), eosinophil cationic protein (ECP), and eosinophil-derived neurotoxin (EDN; Gleich and Adolphson, 1986; Kita, 1996]. These mediators are generally considered to be involved in the eosinophil-mediated defense

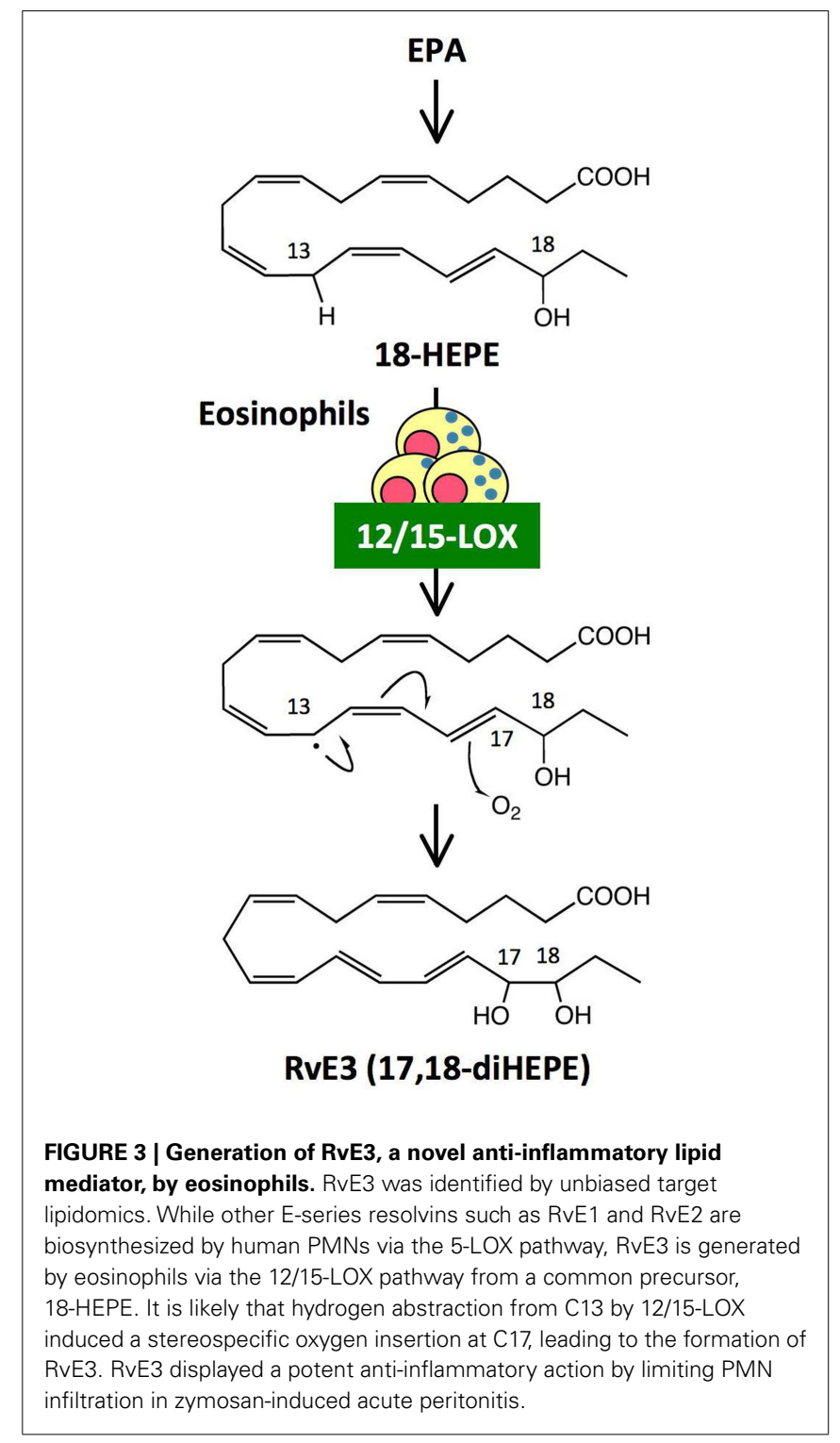

against parasitic infections. Also, locally accumulated eosinophils are considered to be involved in the pathogenesis of allergic diseases such as asthma. Recently, eosinophils were shown to promote alternatively activated macrophages in an IL-4 and IL-13 dependent manner, which in turn improved glucose metabolism (Wu et al., 2011). Eosinophils in the resolution phase may modulate macrophage phenotype, and thereby promote resolution of acute inflammation.

\section{A NOVEL EOSINOPHIL-DERIVED LIPID MEDIATOR WITH ANTIIINFLAMMATORY PROPERTIES}

Omega-3 PUFAs such as EPA and DHA are enriched in fish oils, and have beneficial effects in many inflammatory disorders including cardiovascular disease, arthritis, colitis, and asthma. Omega-3 PUFAs have been proposed to act via several mechanisms, such as by preventing conversion of omega- 6 PUFA arachidonic acid to pro-inflammatory eicosanoids, and by being converted to potent anti-inflammatory mediators such 


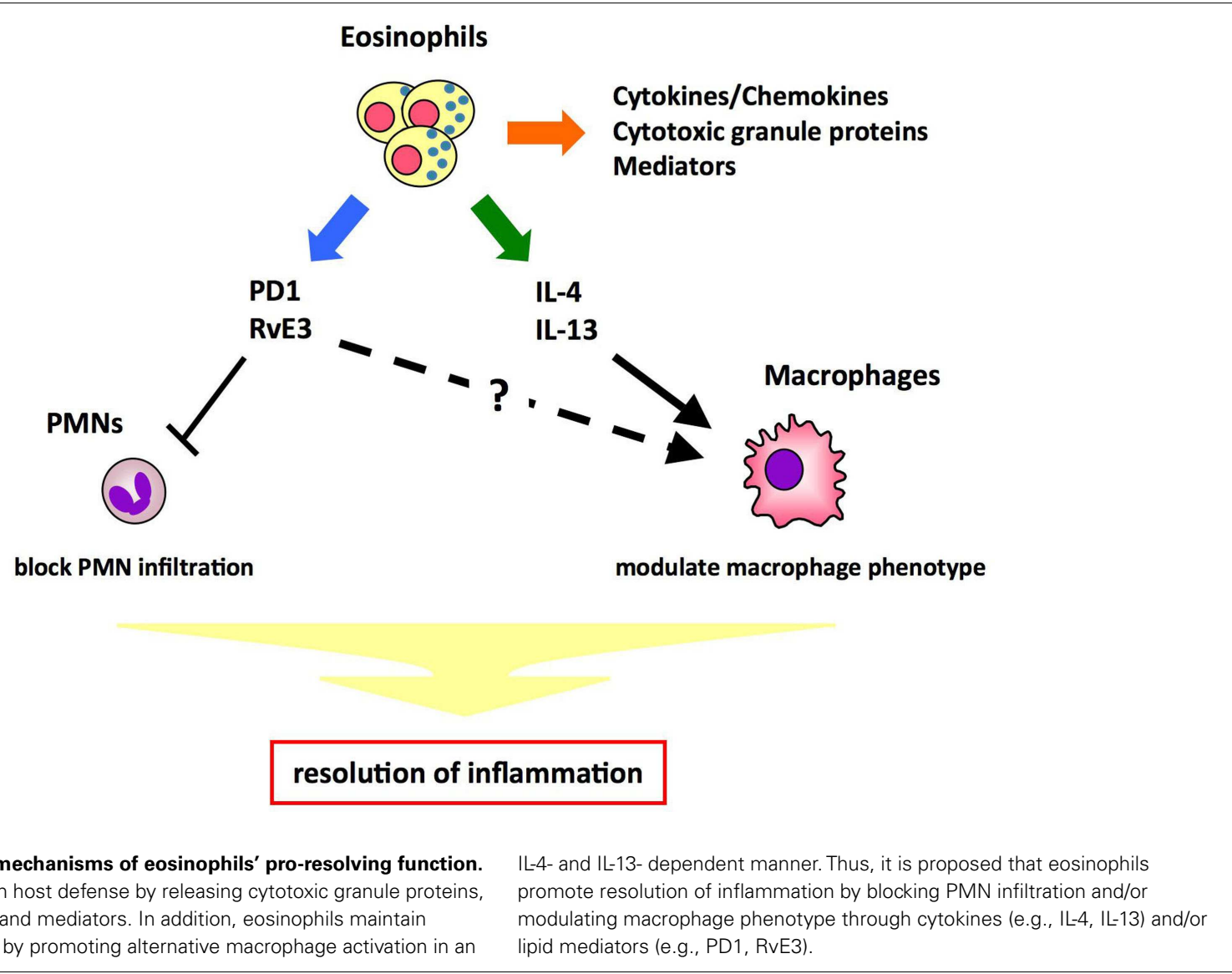

as resolvins. RvE1 [5S,12R,18R-trihydroxy-eicosapentaenoic acid (EPE)] and RvE2 (5S,18R-dihydroxy-EPE) are biosynthesized by human PMNs via the 5-LOX pathway from a common precursor, 18-hydroxy eicosapentaenoic acid (18-HEPE; Serhan et al., 2000; Arita et al., 2005; Tjonahen et al., 2006; Oh et al., 2011). Analyses by unbiased target lipidomics using LC-MS/MS recently showed that eosinophils converted 18-HEPE into novel 8,18-dihydroxyEPE (8,18-diHEPE), 11,18-diHEPE, 12,18-diHEPE, and 17,18diHEPE (Isobe et al., 2012). Among those, 17,18-diHEPE, termed RvE3, displayed potent anti-inflammatory activity by blocking PMN infiltration in acute peritonitis. Unlike RvE1 and E2, both of which are biosynthesized by PMNs via the 5-LOX pathway, RvE3is biosynthesized via the 12/15-LOX pathway, which is highly expressed in eosinophils (Isobe et al., 2012; Figure 3). As mentioned above, eosinophils are recruited to the inflamed loci and promote resolution of inflammation. Therefore, RvE3 may, at least in part, contribute to the eosinophils' function to regulate acute inflammation and resolution. 12/15-LOX is also expressed in tissue resident macrophages, dendritic cells, mast cells, and airway epithelial cells (Kühn and O'Donnell, 2006). 12/15-LOX deficiency leads to progressive atherosclerosis (Merched et al., 2008), exacerbation of arthritis, and inflammatory joint destruction (Krönke et al., 2009), reduced corneal re-epithelialization (Gronert et al., 2005), and a decline of self tolerance (Uderhardt et al., 2012). Cells expressing 12/15-LOX might be involved in regulating inflammatory responses by locally producing lipid mediators such as RvE3.

\section{PERSPECTIVES}

Eosinophils are known to be involved in allergic diseases and host protection against parasites through the release of cytokines/chemokines, mediators, and cytotoxic products. Here we provide the first evidence that eosinophils act as specific proresolving cells that are recruited and switched on during the resolution phase of acute peritonitis. Resolution of inflammation is a highly regulated and coordinated process that involves the suppression of PMN migration, macrophage recruitment, phagocytosis and clearance of apoptotic cells, and tissue debris. In innate immune responses, the macrophage phenotype is critical in determining whether the inflamed site resolves or progresses to chronic inflammation. Resolution phase macrophages express markers such as mannose receptor (MMR) and CD36, which are typical of alternatively activated macrophages (Fernandez-Boyanapalli et al., 2010). A recent study indicates that eosinophils promote alternative macrophage activation in an IL-4 and IL-13 dependent manner (Wu et al., 2011). Therefore, it is likely that eosinophils promote resolution of inflammation by blocking PMN infiltration and/or modulating macrophage phenotype through cytokines and/or lipid mediators (Figure 4). Detailed characterization of eosinophils in the resolution phase will provide insights into the molecular mechanisms for resolution of inflammation.

Failure of acute inflammation to adequately resolve might contribute to the development of chronic inflammation and tissue dysfunction. Indeed, some of the most common and difficult to treat diseases are linked to excessive, uncontrollable, or 
chronic inflammation, including cardiovascular disease, rheumatoid arthritis, periodontal disease, asthma, diabetes, and inflammatory bowel disease (IBD), as well as neurological disorders such as Alzheimer's disease and age-related macular degeneration (AMD).

\section{REFERENCES}

Ariel, A., Fredman, G., Sun, Y.-P., Kantarci, A., Van Dyke, T. E., Luster, A. D., and Serhan, C. N. (2006). Apoptotic neutrophils and $\mathrm{T}$ cells sequester chemokines during immune response resolution via modulation of CCR5 expression. Nat. Immunol. 7, 1209-1216.

Arita, M., Bianchini, F., Aliberti, J., Sher, A., Chiang, N., Hong, S., Yang, R., Petasis, N. A., and Serhan, C. N. (2005). Stereochemical assignment, antiinflammatory properties, and receptor for the omega-3 lipid mediator resolvin E1. J. Exp. Med. 201, 713-722.

Bannenberg, G., and Serhan, C. N. (2010). Specialized pro-resolving lipid mediators in the inflammatory response: an update. Biochim. Biophys. Acta 1801, 1260-1273.

Bannenberg, G. L., Chiang, N., Ariel, A., Arita, M., Tjonahen, E., Gotlinger, K. H., Hong, S., and Serhan, C. N. (2005). Molecular circuits of resolution: formation and actions of resolvins and protectins. J. Immunol. 174, 4345-4355.

Blaho, V. A., Buczynski, M. W., Brown, C. R., and Dennis, E. A. (2009). Lipidomic analysis of dynamic eicosanoid responses during the induction and resolution of lyme arthritis. J. Biol. Chem. 284, 21599-21612.

Chiang, N., Fredman, G., Bäckhed, F., Oh, S. F., Vickery, T., Schmidt, B. A., and Serhan, C. N. (2012). Infection regulates pro-resolving mediators that lower antibiotic requirements. Nature 484, 524-528.

Colgan, S. P., Serhan, C. N., Parkos, C. A., Delp-Archer, C., and Madara, J. L. (1993). Lipoxin A4 modulates transmigration of human neutrophils across intestinal epithelial monolayers. J. Clin. Invest. 92, 75-82.

Corry, D. B., Folkesson, H. G., Warnock, M. L., Erle, D. J., Matthay, M. A., Wiener-Kronish, J. P., and Locksley, R. M. (1996). Interleukin 4, but not interleukin 5 or eosinophils, is required in a murine model of acute airway hyperreactivity. J. Exp. Med. 183, 109-117.

Fernandez-Boyanapalli, R., Frasch, S. C., Riches, D. W., Vandivier, R. W., Henson, P. M., and Bratton, D. L. (2010). $\operatorname{PPAR} \gamma$ activation normalizes resolution of acute sterile inflammation in murine chronic granulomatous disease. Blood 116, 4512-4522.
Gilbert, N. C., Rui, Z., Neau, D. B., Waight, M. T., Bartlett, S. G., Boeglin, W. E., Brash, A. R., and Newcomer, M. E. (2012). Conversion of human 5-lipoxygenase to a 15-lipoxygenase by a point mutation to mimic phosphorylation at Serine-663. FASEB J. 26, 3222-3229.

Gilroy, D. W., Lawrence, T., Perretti, M., and Rossi, A. G. (2004). Inflammatory resolution: new opportunities for drug discovery. Nat. Rev. Drug Discov. 3, 401-416.

Gleich, G. J., and Adolphson, C. R. (1986). The eosinophilic leukocyte: structure and function. $A d v$. Immunol. 39, 177-253.

Godson, C., Mitchell, S., Harvey, K., Petasis, N. A., Hogg, N., and Brady, H. R. (2000). Cutting edge: lipoxins rapidly stimulate nonphlogistic phagocytosis of apoptotic neutrophils by monocytederived macrophages. J. Immunol. 164, 1663-1667.

Gronert, K., Maheshwari, N., Khan, N., Hassan, I. R., Dunn, M., and Laniado Schwartzman, M. (2005). A role for the mouse 12/15-lipoxygenase pathway in promoting epithelial wound healing and host defense. J. Biol. Chem. 280, 15267-15278.

Hong, S., Gronert, K., Devchand, P., Moussignac, R.-L., and Serhan, C. N. (2003). Novel docosatrienes and 17S-resolvins generated from docosahexaenoic acid in murine brain, human blood and glial cells: autacoids in antiinflammation. J. Biol. Chem. 278, 14677-14687.

Isobe, Y., Arita, M., Matsueda, S., Iwamoto, R., Fujihara, T., Nakanishi, H., Taguchi, R., Masuda, K., Sasaki, K., Urabe, D., Inoue, M., and Arai, H. (2012). Identification and structure determination of novel anti-inflammatory mediator resolvin E3, 17,18dihydroxyeicosapentaenoic acid. J. Biol. Chem. 287, 10525-10534.

Kita, H. (1996). The eosinophil: a cytokine-producing cell? J. Allergy Clin. Immunol. 97, 889-892.

Krönke, G., Katzenbeisser, J., Uderhardt, S., Zaiss, M. M., Scholtysek, C., Schabbauer, G., Zarbock, A., Koenders, M. I., Axmann, R., Zwerina, J., Baenckler, H. W., van den Berg, W., Voll, R. E., Kühn, H., Joosten, L. A., and Schett, G. (2009). 12/15-Lipoxygenase counteracts

The studies summarized here raise the possibility that modulating eosinophil number and function, or using endogenous mediators released from eosinophils in the resolution phase could provide novel therapies for many inflammatory diseases.

inflammation and tissue damage in arthritis. J. Immunol. 183 3383-3389.

Kühn, H., and O’Donnell, V. B. (2006). Inflammation and immune regulation by 12/15-lipoxygenases. Prog. Lipid Res. 45, 334-356.

Larsen, G. L., and Henson, P. M. (1983). Mediators of inflammation. Annu. Rev. Immunol. 1, 335-359.

Levy, B. D., Clish, C. B., Schmidt, B., Gronert, K., and Serhan, C. N. (2001). Lipid mediator class switching during acute inflammation: signals in resolution. Nat. Immunol. 2, 612-619.

Marcheselli, V. L., Hong, S., Lukiw, W. J., Tian, X. H., Gronert, K., Musto, A., Hardy, M., Gimenez, J. M., Chiang, N., Serhan, C. N., and Bazan, N. G. (2003). Novel docosanoids inhibit brain ischemia-reperfusionmediated leukocyte infiltration and pro-inflammatory gene expression. J. Biol. Chem. 278, 43807-43817.

Merched, A. J., Ko, K., Gotlinger, K. H., Serhan, C. N., and Chan, L. (2008). Atherosclerosis. Evidence for impairment of resolution of vascular inflam- mation governed by specific lipid mediators. FASEB J. 22, 3595-3606.

Oh, S. F., Pillai, P. S., Recchiuti, A., Yang, R., and Serhan, C. N. (2011). Pro-resolving actions and stereoselective biosynthesis of $18 \mathrm{~S}$ E-series resolvins in human leukocytes and murine inflammation. J. Clin. Invest. 121, 569-581.

Schwab, J. M., Chiang, N., Arita, M., and Serhan, C. N. (2007) Resolvin E1 and protectin D1 activate inflammation-resolution programmes. Nature 447, 869-874.

Serhan, C. N. (1997). Lipoxins and novel aspirin-triggered 15-epilipoxins (ATL): a jungle of cell-cell interactions or a therapeutic opportunity? Prostaglandins 53 107-137.

Serhan, C. N. (2005). Special issue on lipoxins and aspirin-triggered lipoxins. Prostaglandins Leukot. Essent. Fatty Acids 73, 139-321.

Serhan, C. N. (2007). Resolution phase of inflammation: novel endogenous anti-inflammatory and proresolving lipid mediators and pathways. Annu. Rev. Immunol. 25, 101-137.

Serhan, C. N., Clish, C. B., Brannon, J., Colgan, S. P., Chiang, N., and Gronert, K. (2000). Novel functional sets of lipid-derived mediators with antiinflammatory actions generated from omega-3 fatty acids via cyclooxygenase 2-nonsteroidal antiinflammatory drugs and transcellular processing. J. Exp. Med. 192, 1197-1204.

Serhan, C. N., Dalli, J., Karamnov, S., Choi, A., Park, C. K., Xu, Z. Z., Ji, R. R., Zhu, M., and Petasis, N. A. (2012). Macrophage proresolving mediator maresin 1 stimulates tissue regeneration and controls pain. FASEB J. 26, 1755-1765.

Serhan, C. N., Gotlinger, K., Hong, S., Lu, Y., Siegelman, J., Baer, T., Yang, R., Colgan, S. P., and Petasis, N. A. (2006). Anti-inflammatory actions of neuroprotectin D1/protectin D1 and its natural stereoisomers: assignments of dihydroxy-containing docosatrienes. J. Immunol. 176 , 1848-1859.

Serhan, C. N., Hong, S., Gronert, K., Colgan, S. P., Devchand, P. R., Mirick, G., and Moussignac, R.-L. (2002). Resolvins: a family of bioactive products of omega-3 fatty acid transformation circuits initiated by aspirin treatment that counter proinflammation signals. J. Exp. Med. 196, 1025-1037.

Serhan, C. N., and Savill, J. (2005). Resolution of inflammation: the beginning programs the end. Nat. Immunol. 6, 1191-1197.

Serhan, C. N., Yang, R., Martinod, K., Kasuga, K., Pillai, P. S., Porter, T. F., Oh, S. F., and Spite, M. (2009). Maresins: novel macrophage mediators with potent antiinflammatory and proresolving actions. J. Exp. Med. 206, 15-23.

Spite, M., Norling, L. V., Summers, L., Yang, R., Cooper, D., Petasis, N. A., Flower, R. J., Perretti, M., and Serhan, C. N. (2009). Resolvin D2 is a potent regulator of leukocytes and controls microbial sepsis. Nature 461, 1287-1291.

Sun, Y. P., Oh, S. F., Uddin, J., Yang, R., Gotlinger, K., Campbell, E., Colgan, S. P., Petasis, N. A., and Serhan, C. N. (2007). Resolvin $\mathrm{D} 1$ and its aspirin-triggered 17R epimer. Stereochemical assignments, anti-inflammatory properties, and enzymatic inactivation. J. Biol. Chem. 282, 9323-9334.

Tjonahen, E., Oh, S. F., Siegelman, J., Elangovan, S., Percarpio, K. B., Hong, S., Arita, M., and Serhan, C. N. (2006). Resolvin E2: identification 
and anti-inflammatory actions. Pivotal role of human 5-lipoxygenase in resolvin E series biosynthesis. Chem. Biol. 13, 1193-1202.

Uderhardt, S., Herrmann, M., Oskolkova, O. V., Aschermann, S., Bicker, W., Ipseiz, N., Sarter, K., Frey, B., Rothe, T., Voll, R., Nimmerjahn, F., Bochkov, V. N., Schett, G., and Krönke, G. (2012). 12/15-lipoxygenase orchestrates the clearance of apoptotic cells and maintains immunologic tolerance. Immunity 36, 1-13.
Wu, D., Molofsky, A. B., Liang, H. E., Ricardo-Gonzalez, R. R., Jouihan, H. A., Bando, J. K., Chawla, A., and Locksley, R. M. (2011). Eosinophils sustain adipose alternatively activated macrophages associated with glucose homeostasis. Science 332, 243-247.

Yamada, T., Tani, Y., Nakanishi, H., Taguchi, R., Arita, M., and Arai, H. (2011). Eosinophils promote resolution of acute peritonitis by producing proresolving mediators in mice. FASEB J. 25, 561-568.
Conflict of Interest Statement: The authors declare that the research was conducted in the absence of any commercial or financial relationships that could be construed as a potential conflict of interest.

Received: 18 July 2012; accepted: 07 August 2012; published online: 28 August 2012.

Citation: Isobe $Y$, Kato $T$ and Arita $M$ (2012) Emerging roles of eosinophils and eosinophil-derived lipid mediators in the resolution of inflammation. Front. Immun. 3:270. doi 10.3389/fimmu.2012.00270

This article was submitted to Frontiers in Inflammation, a specialty of Frontiers in Immunology.

Copyright () 2012 Isobe, Kato and Arita. This is an open-access article distributed under the terms of the Creative Commons Attribution License, which permits use, distribution and reproduction in other forums, provided the original authors and source are credited and subject to any copyright notices concerning any third-party graphics etc. 